\title{
ACPR and IM3R Correlation of Broadband Signals in Microwave and Millimeter Wave Frequencies
}

\author{
Wei-Chien Chen, Jeng-Han Tsai, Shih-Yu Chen, and Tian-Wei Huang \\ Department of Electrical Engineering and Graduate Institute of Communication Engineering, \\ National Taiwan University, Taipei, Taiwan 106, R.O.C., Tel: +886-2-23635251-342 \\ Email: twhuang@cc.ee.ntu.edu.tw
}

\begin{abstract}
In this paper, we correlate the two-tone test data, third order inter-modulation distortion ratio (IM3R) with the ACPR from multi-tone test for broadband signals at $1.95 \mathrm{GHz}$ and $2.4 \mathrm{GHz}$. We also up-convert these broadband signals to millimeter-wave frequency to verify the correlation equation for $44-\mathrm{GHz}$ power amplifiers. These measurement results show good agreement, within $\pm 1 \mathrm{~dB}$, between two-tone and multi-tone tests up to the $P_{1 \mathrm{~dB}}$ point. It is observed that, as the input power increased, the multi-tone ACPR has a similar trend as the ACPR for broadband signals, like WCDMA and 16-QAM in microwave and millimeter-wave frequency band.
\end{abstract}

Index Terms - Communication system nonlinearities, ACPR, IM3R, Millimeter-wave.

\section{INTRODUCTION}

With the fast development of cellular and personal wireless communication system, such as IS-95 CDMA, WCDMA, IEEE $802.11 \mathrm{~g}$, and etc., microwave frequency band have become crowded with various applications. Therefore the need to enhance spectral efficiency is an urgent issue. To utilize the limited bandwidth effectively, modern digital communication systems tend to use complex digital modulation schemes which have stringent linearity specifications of the power amplifier to reduce the spectral re-growth.

ACPR measurements represent the industry standard in linearity of power amplifiers. Due to the expensive cost and complex excitation of the digital-modulated signals, investigations and calculations of linearity have been made to get results closer to the system's final operation regime with two-tone test. The most popular way is finding a relationship between third order intermodulation distortion ratio (IM3R) and ACPR. It has been found that ACPR and IM3R have correlation under $2 \mathrm{GHz}$ with bandwidth of $1.2288 \mathrm{MHz}$ [1]. Other works involve with the relationship between two-tone test IM3R and multi-tone test ACPR to approach the digital modulated signals [2]-[4]. Also, another equation for CDMA signal has been shown in narrower bandwidth under $2 \mathrm{GHz}$ [5] [6].

In this paper, we verified that the correlated equation between multi-tone ACPR and measured IM3R still can be used in broadband signal, such as $3.84 \mathrm{MHz}$ main channel bandwidth for WCDMA and $16 \mathrm{MHz}$ main channel bandwidth centered for IEEE 802.11g. Furthermore the correlated equation can be applied in millimeter-wave frequency band. By using two-tone test and correlated equation, the ACPR of the millimeterwave power amplifier can be calculated simply without generating digital modulation signal in millimeter-wave.

\section{DISCRIPTION}

For characterization of nonlinear system or devices such as power amplifier, multi-tone carriers generated from sinewaves, typically with constant tone spacing is an adequate extension of the use of two-tone signals for estimating the nonlinear distortion products. Therefore, we start our analysis by exciting multi-tone carrier signal.

And, the correlated equation between multi-tone ACPR and measured IM3R obtained from a conventional twotone test $\left(\mathrm{IM}^{2} \mathrm{R}_{2 \text {-tone }}\right)$ was derived in [2]. This paper assumed that the nonlinear system is a memoryless third order microwave circuit. The final equation is shown as

$$
A C P R_{\text {multitone }}=I M 3 R_{2-\text { tone }}+10 \log \left\{\frac{n^{3}}{4 A+B}\right\}-6
$$

Here, $n$ represents the number of multitone carriers in main power band, and $A$ and $B$ which are both function of $n$ represent the modified terms defined in [2].

\section{BROADBAND SIGNAL ACPR IN LOW FREQURNCY}

In order to be sure of the correlated equation still can be used to replace measured ACPR from multi-tone signal with measured IM3R in broadband signal; we started the measurement test with $3.84 \mathrm{MHz}$ main channel bandwidth centered at $1.95 \mathrm{GHz}$ for WCDMA and with $16 \mathrm{MHz}$ main channel bandwidth centered at $2.4 \mathrm{GHz}$ for 16-QAM digital modulation signal.

The two-tone signal and multi-tone carriers were generated by Agilent E4438C ESG Vector Signal Generator. The device under test (DUT) is MAXIM power amplifier evaluation kit MAX2247.The measurement setup is shown in Fig. 1. 




Fig. 1. Multitone intermodulation distortion spectrum measurement setup in low frequency.

First, we chose the two-tone frequency spacing equals to $100 \mathrm{kHz}$ (the square marked one) to measure the IM3R. Next, we restricted multi-tone sine wave component placement to a $60 \mathrm{kHz}$ grid in $3.84 \mathrm{MHz}$ to approximate WCDMA signal and $250 \mathrm{kHz}$ grid in $16 \mathrm{MHz}$ to equal the digital modulated signal 16-QAM. This restriction cause the number of multi-tone to six: $n=3,5,9,17,33$, 65 (64 for Agilent E4438C maximum tone number).

As we started the multi-tone test increasing from $n=3$ to $n=64$, we found that the accuracy of the equation in calculated ACPR to measured ACPR is also increasing, which agrees with data from [2]. Therefore, we take the 64-tones as an illustration.

Calculating the two intermediate values with $n=64$ in equation (1) gives:

$$
A C P R_{\text {multitone }}=I M 3 R_{2-\text { tone }}-1.17652
$$

Use equation (2) to calculate the multi-tone ACPR with measured IM3 $\mathrm{R}_{2 \text {-tone }}$ from two-tone test and measure the ACPR generated from multi-tone, and modulated signal, then we can have the following comparison results which are shown in Fig. 2. Note that we herein measured ACPR by the ratio of upper bond adjacent channel and main power channel.

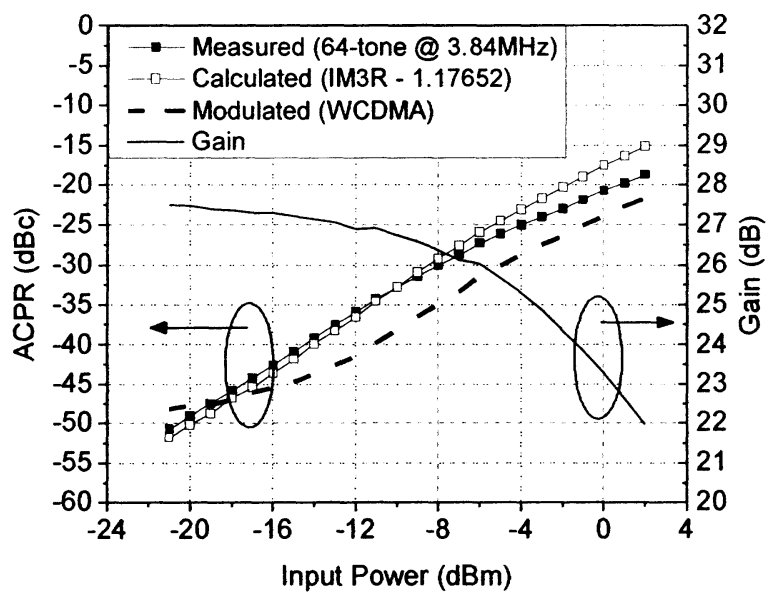

(a)

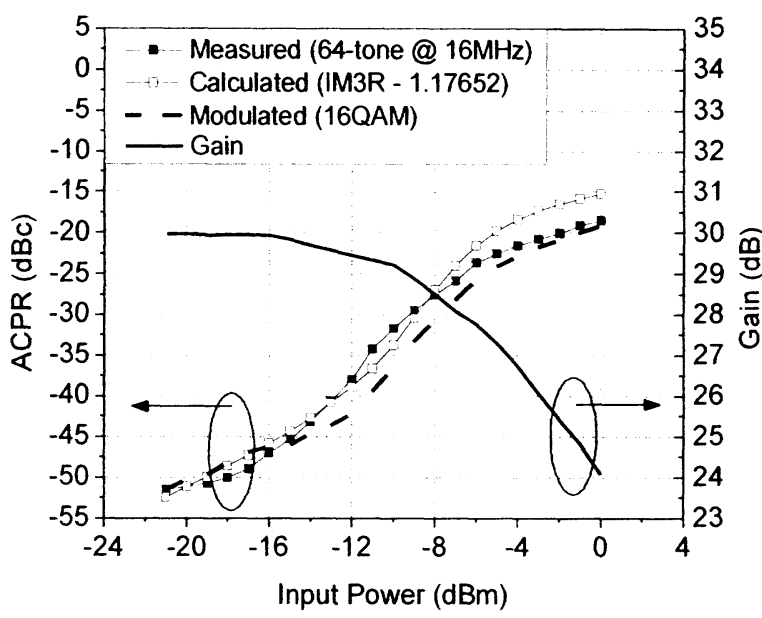

(b)

Fig. 2. Verification between measured and calculated ACPR for broadband signal with 64 tones in low frequency.(a) WCDMA (b) 16-QAM.

From the above figure, we found that there is a deviation less than $\pm 1 \mathrm{~dB}$ under the $P_{1 \mathrm{~dB}}$ point between measured ACPR and calculated ACPR from equation (2) for broadband signal. As gain falls to the $\mathrm{P}_{1 \mathrm{~dB}}$ point and near the saturation region, the calculated results have a little difference with the measured results. This is because near $1 \mathrm{~dB}$ gain compression point, the assumption of equation (2) does not agree with the real situation because of the higher order nonlinear distortions need to be considered.

\section{IMPLEMENTATION AND MEASUREMENT IN MILLIMETER WAVE FREQUENCY}

The millimeter-wave measured instruments and devices list as follow: we used Agilent E8247C PSG as a local oscillator (LO), Agilent E4438C ESG as a baseband to intermediate frequency (IF) vector signal generator, Wisewave Module FDS-2F210812-01 as a sub-harmonic pumped mixer, Wisewave Power Module AHP42042530-01 as a driver amplifier to drive the power amplifier we need, QuinStar Power Module QGW38481728-JO as a device under test (DUT), and Agilent E4448A PSA as the spectrum analyzer. The millimeter wave multi-tone spectrum measurement setup is shown in Fig. 3.

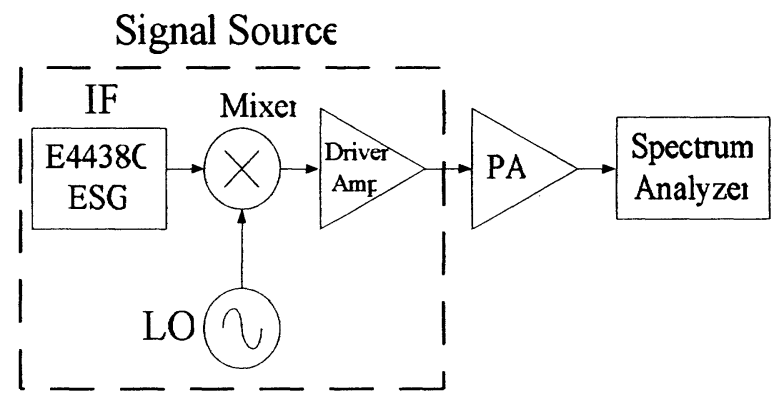

Fig. 3. Multi-tone inter-modulation spectrum measurement setup in millimeter wave frequencies 
We set the millimeter-wave center frequency at 44 $\mathrm{GHz}$ to be our test frequency. Hence, we chose LO at 24 $\mathrm{GHz}, \mathrm{IF}$ at $4 \mathrm{GHz}$ and used sub-harmonic mixer to mix the IF signal to $44 \mathrm{GHz}$. Because of the conversion loss in sub-harmonic mixer and cable loss at such high frequency, we need a driver amplifier with high gain and power to drive the DUT (PA) we need.

The measurement ACPR from multi-tone carrier with 64 tones and the calculated ACPR used the same equation (2) for broadband signal $3.84 \mathrm{MHz}$ (WCDMA) and $16 \mathrm{MHz}$ (16-QAM) are shown in Fig. 4(a) and Fig. 4(b), respectively.

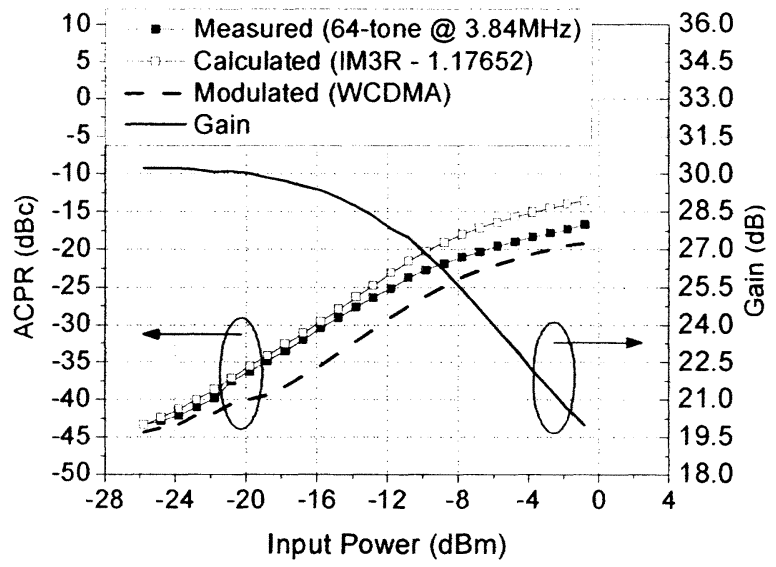

(a)

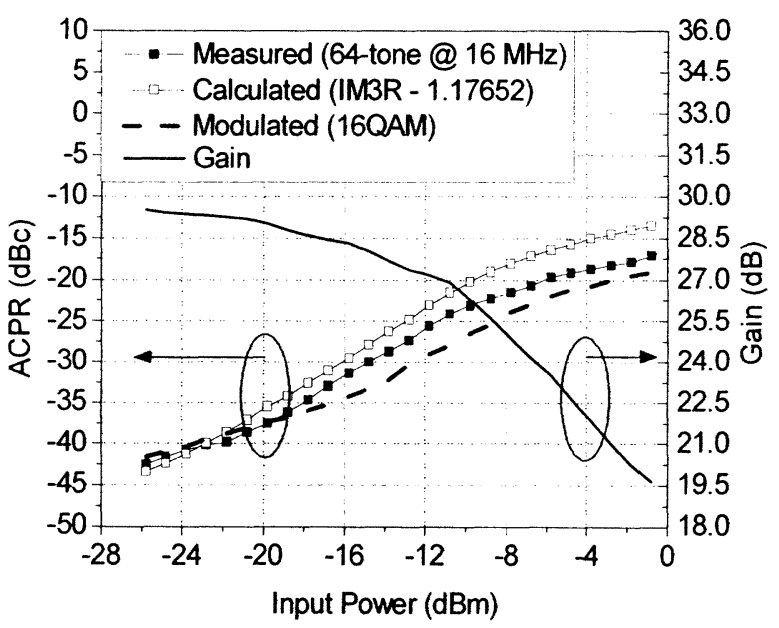

(b)

Fig. 4. Comparison between measured and calculated ACPR for broadband signal with 64 tones at $44 \mathrm{GHz}$ millimeter wave frequency. (a) WCDMA (b) 16-QAM

Comparing the measurement results in low frequency (Fig. 2) and in millimeter wave frequency (Fig. 4), we found that the precise region also up to the $P_{1 d B}$ point with the tolerance around $\pm 1 \mathrm{~dB}$ due to measurement deviation in millimeter-wave frequency.

We also found that as the signal bandwidth get wider (Fig. 4(a) and Fig. 4(b)), the difference in the linear region (below $1 \mathrm{~dB}$ compression point) between calculated results and measured results will become larger.
From Fig. 2 and Fig. 4, we showed that the ACPR of the digital modulation signal, such as WCDMA, 16QAM has the similar trend with the multi-tone carrier inter-modulation component in low frequency and in millimeter wave frequency, but it is overestimated by approximately $5 \mathrm{~dB}$ near the $\mathrm{P}_{1 \mathrm{~dB}}$ point. This comparison result has good agreement with narrower band signal data from [2].

\section{CONCLUSION}

In conclusion, this paper we verified the relationship between IM3R and multi-tone ACPR can be used for broadband signal up to $16 \mathrm{MHz}$ power channel bandwidth at $44 \mathrm{GHz}$ millimeter wave frequencies. And as the power channel bandwidth goes wider; the difference between IM3R and ACPR from multi-tone will becomes larger. We also showed that the correlation between multi-tone ACPR and ACPR of broadband signal in microwave and millimeter wave frequencies has similar trend but is still overestimated.

\section{ACKNOWLEDGEMENT}

The work was supported in part by National Science Council (NSC 93-2219-E-002-025 and NSC 92-2220-E002-005).

\section{REFERENCES}

[1] W. Xinwei, H. Nakamura, and R. Singh, "ACPR, IM3 and their correlation for PCS CDMA power amplifier," in 50th ARFTG Dig., Dec. 1997, pp.91-96.

[2] K. Remley, "Multisine excitation for ACPR measurements," in IEEE MTT-S Int. Microwave Symp. Dig., Philadelphia, PA, Jun. 2003 [CD ROM], pp. 21412144.

[3] Pedro, J. C., and N. B. De Carvalho, "On the use of multitone techniques for assessing RF components' intermodulation distortion," IEEE Trans .on Microwave Theory and Techniques, vol. 47, No. 12, December 1999, pp. 2393-2402.

[4] Carvalho, N. B. and J. C. Pedro, "Multi-tone intermodulation distortion performance of 3rd order microwave circuits," IEEE Symposium on Microwave Theory and Techniques, Anaheim, USA, vol. 2, June 1999, pp. 763-766.

[5] Jin-Su Ko; Jeong-Keun Kim; Beom-Kyu Ko; Dong-Bin Cheon; Byeong-Ha Park, "Enhanced ACPR technique by class AB in PCS driver amplifier," VLSI and CAD, 1999. ICVC '99. 6th International Conference on , 26-27, Oct. 1999, pp. $376-379$.

[6] Goh, T.S.L.; Pollard, R.D, "ACPR prediction of CDMA systems through statistical behavioural modelling of power amplifiers with memory," 2002 The 8th International Conference on Communication Systems, vol. 2, Nov. 2002, pp. $1189-1193$.

[7] Peter B. Kenignton, High-Linearity RF Amplfier Design, Artech House, Boston, 2000.

[8] MAXIM power amplifier MAX2247 data sheet 